\title{
High expression of P21-activated kinase 5 protein is associated with poor survival in gastric cancer
}

\author{
TOMOKI ABURATANI ${ }^{1}$, MIKITO INOKUCHI ${ }^{1}$, YOKO TAKAGI ${ }^{2}$, TOSHIAKI ISHIKAWA ${ }^{2}$, \\ KEISUKE OKUNO $^{1}$, KENTARO GOKITA ${ }^{1}$, CHIHARU TOMII ${ }^{1}$, TOSHIRO TANIOKA ${ }^{1}$, \\ HIDEAKI MURASE ${ }^{1}$, SHO OTSUKI ${ }^{1}$, HIROYUKI UETAKE ${ }^{2}$, KAZUYUKI KOJIMA ${ }^{1,3}$ and TATSUYUKI KAWANO ${ }^{1}$ \\ Departments of ${ }^{1}$ Gastrointestinal Surgery and ${ }^{2}$ Surgical Specialty; \\ ${ }^{3}$ Center for Minimally Invasive Surgery, Tokyo Medical and Dental University, Tokyo 113-8519, Japan
}

Received August 31, 2016; Accepted March 3, 2017

DOI: $10.3892 / \mathrm{ol} .2017 .6115$

\begin{abstract}
P21-activated kinase 5 (PAK5), also termed PAK7, is one of the six members of the PAK family of serine/threonine kinases, which are downstream effectors in several cancer signaling pathways. PAK5 promotes neural outgrowth, contributes to microtubule stability and induces resistance to apoptosis. However, the clinical importance of PAK5 in gastric cancer has not been comprehensively investigated. In the present study, PAK5 expression was evaluated in gastric cancer tissue samples. Furthermore, the associations between high expression of PAK5, and clinicopathological features and prognosis were examined. PAK 5 expression in primary gastric cancer specimens resected from 279 patients who underwent gastrectomy at the Tokyo Medical and Dental University Hospital was evaluated using immunohistochemistry. Of the 279 patients, 44 (15.8\%) exhibited high PAK5 expression, which was significantly associated with the differentiated pathological type (differentiated vs. undifferentiated; $\mathrm{P}<0.001$ ), depth of tumor invasion (T1 vs. T2-T4; $\mathrm{P}<0.001$ ), lymph node metastasis (N0 vs. N1-N3; $\mathrm{P}<0.001$ ), presence of distant metastasis or recurrence (present vs. absent; $\mathrm{P}=0.038$ ), advanced tumor stage (I vs. II-IV; $\mathrm{P}=0.001$ ) and worse disease-specific survival $(\mathrm{P}=0.013)$. In stage I-III disease, 38/254 (15.0\%) patients exhibited high PAK5 expression, and high expression of PAK5 was significantly associated with relapse-free interval $(\mathrm{P}=0.044)$. PAK5 may serve an important role in tumor progression and influence the outcome of patients with gastric cancer.
\end{abstract}

Correspondence to: $\mathrm{Dr}$ Mikito Inokuchi, Department of Gastrointestinal Surgery, Tokyo Medical and Dental University, 1-5-45 Yushima, Bunkyo-ku, Tokyo 113-8519, Japan

E-mail: m-inokuchi.srg2@tmd.ac.jp

Key words: P21-activated kinase 5, P21-activated kinase 7, gastric cancer, immunohistochemistry, prognosis

\section{Introduction}

Globally, gastric cancer was ranked fifth for cancer incidence (984,000 cases) and second for cancer-associated mortality (841,000 mortalities) rates in 2013 (1). Complete surgical removal of the tumor is the standard treatment for gastric cancer, and it is possible to cure patients with early-stage disease. However, even following macroscopic complete removal of the tumor by standard resection with D2 lymphadenectomy, advanced gastric cancer possesses a poor clinical outcome. Despite recent advances in systemic chemotherapy, an optimal global standard has not yet been established for advanced gastric cancer (2) and there is no internationally recognized standard or preferred regimen for the management of the advanced disease stage. In order to improve outcomes, it is essential to understand the molecular pathogenesis underlying gastric cancer and to identify robust prognostic or predictive biomarkers (3).

P21-activated kinases (PAKs) are a family of serine/threonine kinases that serve as downstream effectors in several cancer signaling pathways. PAKs are overexpressed, hyper-activated or amplified in several types of human tumor, which make them attractive novel therapeutic targets. PAKs are categorized into group I (PAK1, 2 and 3) and group II (PAK4, 5 and 6), based on their amino acid homologies (4-6). PAK5 is the latest PAK family member to be identified and is also termed PAK7 (7-9). PAKs participate in a number of signaling pathways that are commonly deregulated in human cancer cells. PAK1 is a component of the mitogen-activated protein kinase, JUN N-terminal kinase, steroid hormone receptor and nuclear factor $\kappa \mathrm{B}$ signaling pathways, which have all been associated with oncogenesis. Overexpression of PAK1 protein occurs in breast, colon, ovarian and brain cancer, and PAK4 gene amplification and protein overexpression was reported in pancreatic cancer (4).

At the Tokyo Medical and Dental University (Tokyo, Japan), Kobayashi et al (10) revealed that high PAK4 expression was significantly correlated with clinicopathological variables associated with tumor progression. These variables consisted of: Depth of invasion; metastatic lymph nodes; pathological stage; distant metastasis; or recurrent disease. High PAK4 expression was significantly associated with poorer 
disease-specific survival (DSS) $(\mathrm{P}<0.001)$ and relapse-free survival (RFS) $(\mathrm{P}<0.001)(10)$. The role of PAK5 in various types of cancer has been investigated by several groups. Inhibiting PAK5 expression induced a 7-fold increase in apoptosis in the pancreatic adenocarcinoma cell line MiaPaCa-2, suggesting that PAK5 is a kinase that may protect pancreatic cancer cells from apoptosis (11) PAK5 overexpression assessed using immunohistochemistry was reported in certain colorectal cancer types, and PAK5 reduced colorectal carcinoma cell adhesion; however PAK5 promoted cellular migration on collagen type I, indicating that PAK5 is involved in colorectal cancer cell migration and invasion (12). Immunohistochemical analysis demonstrated that PAK5 expression was upregulated significantly in different gastric cancer cell lines and gastric cancer tissue samples, as compared with human embryonic kidney 293 cells and adjacent normal tissue samples (13). The PAK5 mRNA level was significantly higher in 25/30 human hepatocellular carcinoma samples compared with the matched paraneoplastic tissue samples (7). Knockdown of PAK5 inhibited cell proliferation by inducing cell cycle arrest in $\mathrm{G}_{0} / \mathrm{G}_{1}$ phase in human gastric cancer, hepatocellular carcinoma and glioma cells $(13,14)$. PAK5 expression in gastric cancer has not yet been comprehensively investigated in a large sample size.

Using immunohistochemistry, Gu et al (13) examined 57 specimens of human gastric cancer, which consisted of 16 cases of $\mathrm{T} 1$ and $\mathrm{T} 2$ depth of invasion, and the remaining 41 cases of $\mathrm{T} 3$ and $\mathrm{T} 4$. It was concluded that none of the clinicopathological parameters were associated with PAK5 expression. However, according to this study, the expression levels of PAK5 were significantly higher in gastric cancer tissue compared with adjacent normal tissue samples $(\mathrm{P}=0.0001)$. These data suggest that there is a change in PAK5 expression during cancer progression. In the present study, the association between PAK5 expression and clinicopathological factors was investigated, and included a relatively high number of patients with early gastric cancer to investigate whether PAK5 contributes to gastric cancer progression.

\section{Materials and methods}

Patients. Resected specimens from 279 patients, whose mean age was 65 years (range, 21-92 years), with a confirmed pathologic diagnosis of primary gastric cancer, who underwent gastrectomy between January 2003 and December 2008 at the Tokyo Medical and Dental University Hospital, were investigated. Surgery included laparotomy and laparoscopy. There were 213 males and 66 females. Tumors were histopathologically diagnosed based on the 3rd English edition of the Japanese Classification of Gastric Carcinoma (15) guidelines, in which the description of tumor status as denoted by the tumor node metastasis (TNM) stage, is identical to that in the 7th edition of the International Union Against Cancer TNM classification (16).

Papillary and tubular adenocarcinoma were classified as differentiated, while poorly differentiated adenocarcinoma, signet-ring cell carcinoma and mucinous adenocarcinoma were classified as undifferentiated. All patients were followed up every 3-6 months following surgery using serum tumor marker assays and diagnostic imaging using esophagogastroduodenoscopy, computed tomography, ultrasonography or magnetic resonance imaging. Patients with distant metastatic or recurrent disease received chemotherapy with S-1 (tegafur, gimeracil, oteracil potassium; Taiho Pharmaceutical Co., Ltd., Tokyo, Japan) 80-120 mg/day, depending on body surface area, alone or in a combined regimen. The median follow-up was 61 months (2-111 months). A total of 97 (35\%) patients succumbed, including 83 (30\%) who succumbed due to distant metastases or recurrent disease, and 14 (5\%) who succumbed due to other causes.

Ethical approval. All procedures were in accordance with the ethical standards of the institutional review board of Tokyo Medical and Dental University (approval no. 831) and national ethical standards of the responsible committee on human experimentation and with the Helsinki Declaration of 1964, and later versions. Written informed consent was obtained from all patients prior to inclusion in the present study.

Immunohistochemistry. Deparaffinized sections of formalin-fixed paraffin-embedded tissue samples were immunohistochemically stained using the universal Immuno-enzyme Polymer method (Histofine Simple Stain Max Po multikit; Nichirei Co., Tokyo, Japan). An anti-PAK5 polyclonal rabbit antibody (cat. no. ab110069; dilution, 1:150), which was applied in a previous study by Fang et al (7), was purchased from Abcam (Cambridge, UK) and used as the primary antibody diluted with Signal Stain ${ }^{\circledR}$ Antibody Diluent (Cell Signaling Technology, Inc., Danvers, MA, USA). A normal rabbit IgG (cat. no. sc-2027; dilution, 1:1,000; Santa Cruz Biotechnology, Inc., Dallas, TX, USA) was substituted for the primary antibody for the negative controls. Strongly and homogeneously stained gastric adenocarcinoma specimens obtained from the same block were used as positive controls to reduce any bias from staining conditions.

The $4 \mu \mathrm{m}$-thick sections were cut on a microtome, deparaffinized with xylene and rehydrated in a graded ethanol series. Antigen retrieval treatment was performed at $98^{\circ} \mathrm{C}$ in a microwave oven (MI-77; Azumaya, Tokyo, Japan) for $30 \mathrm{~min}$ in $\mathrm{pH}$ 6.0, $10 \mathrm{mmol} / \mathrm{l}$ citrate buffer (Mitsubishi Gas Chemical Company, Inc., Tokyo, Japan). Subsequent to microwaving, the slides were allowed to cool in the staining jar at room temperature until the buffer temperature fell below $45^{\circ} \mathrm{C}$. The slides were subsequently rinsed briefly in PBS and endogenous peroxidase was blocked with 15 min exposure to $3 \%$ hydrogen peroxide in methanol at $22^{\circ} \mathrm{C}$. Following washing with PBS at $22^{\circ} \mathrm{C}$, the slides were incubated with the primary antibody at a 1:150 dilution for 25 min under infrared radiation (MI-77; Azumaya, Tokyo, Japan) at $27^{\circ} \mathrm{C}$. They were then incubated with the second antibody, Histofine Simple Stain Max Po Multi (Nichirei Co.) for $30 \mathrm{~min}$ at $22^{\circ} \mathrm{C}$ and 3,3'-diaminobenzidine tetrahydrochloride solution (Histofine Simple Stain DAB Solution; Nichirei Co.) was applied for color development to visualize the image. Sections were counterstained with $1 \%$ Mayer's hematoxylin (Wako Pure Chemical Industries, Ltd., Osaka, Japan), dehydrated, cleared and mounted.

Interpretation of immunohistochemical data. The slides were separately evaluated by two investigators (TA and YT), who were blinded to patient outcome. The investigators counted whole staining cancer cells of representative cross-sectional 

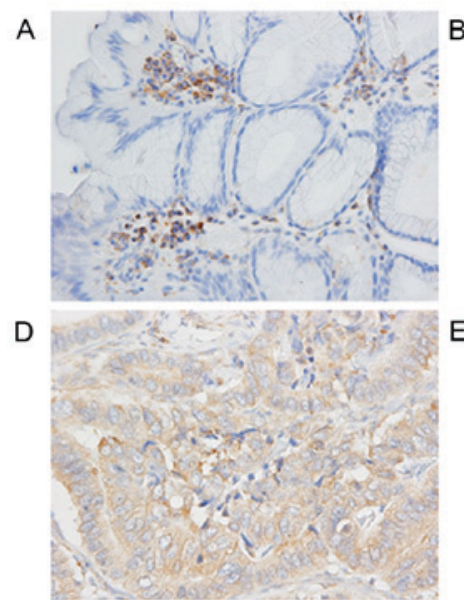

G

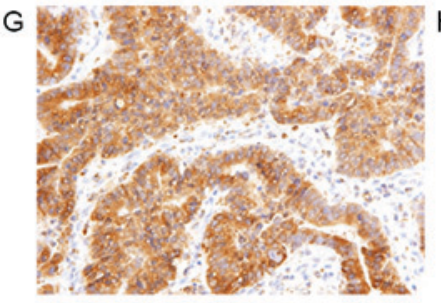

$J$
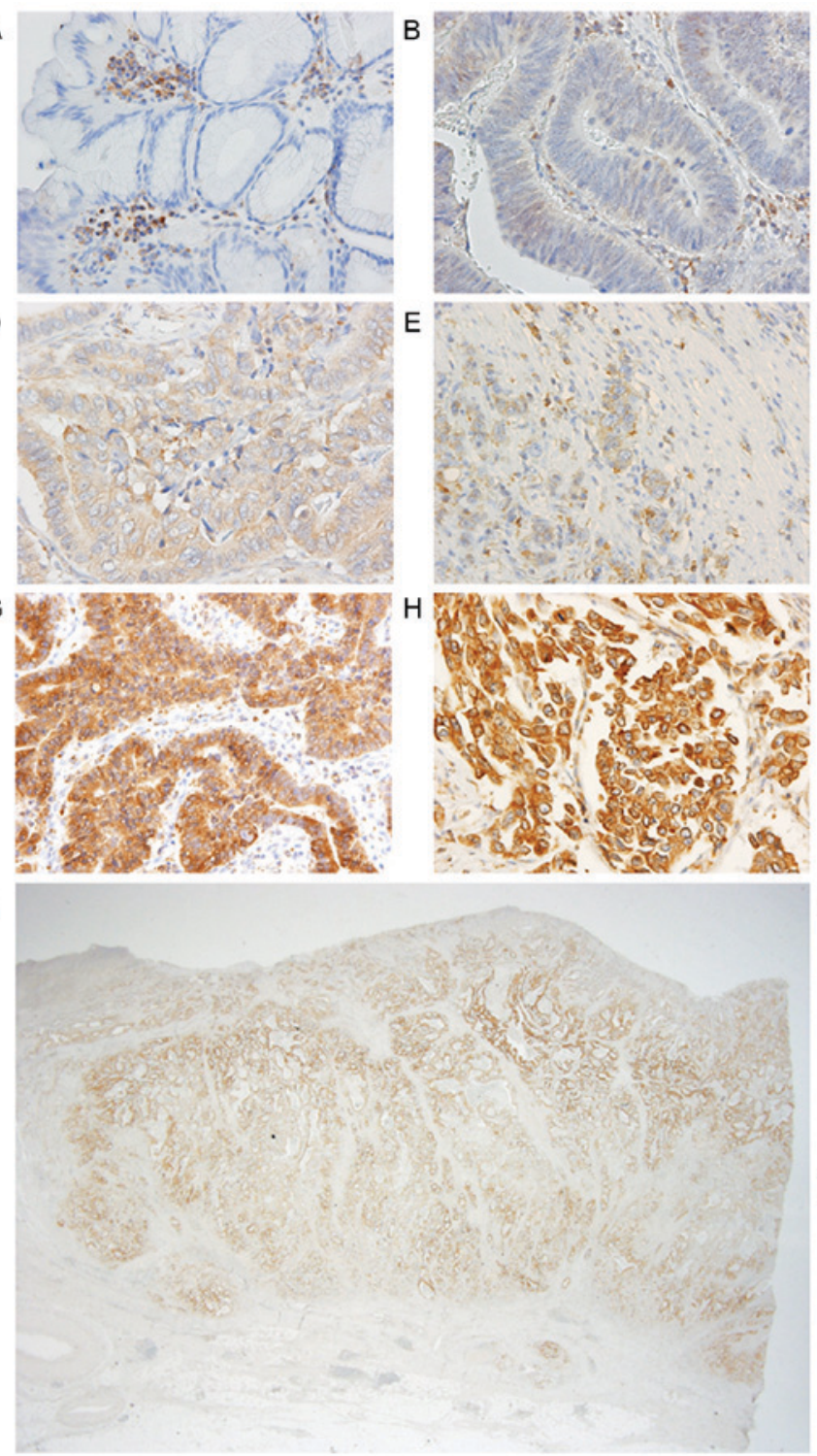

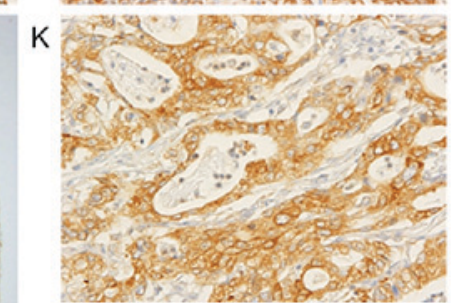

$\mathrm{L}$

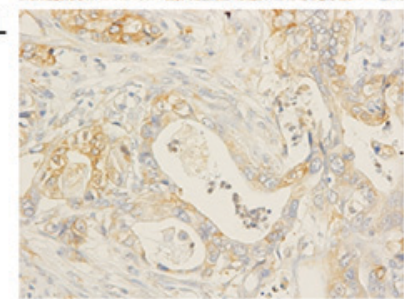

Figure 1. Representative PAK5 staining in normal epithelium and gastric cancer tissue samples. Original magnification, A-I, K, L, x400; J, x12.5. (A) No staining in normal epithelium; (B) very weak staining in differentiated adenocarcinoma; (C) no staining in undifferentiated adenocarcinoma; (D) weak staining in differentiated adenocarcinoma; (E and F) weak staining in undifferentiated adenocarcinoma; $(\mathrm{G})$ strong staining in differentiated adenocarcinoma; (H and I) strong staining in undifferentiated adenocarcinoma; ( $\mathrm{J}$ ) heterogeneous staining of PAK5 in one tumor; (K) strong staining region in ( $\mathrm{J}$ ); (L) weak staining region in $(\mathrm{J})$. PAK5, P21-activated kinase 5.

slices. To evaluate discretely distributed cancer cells, such as in poorly differentiated adenocarcinoma, $\geq$ five fields were counted/section including the most progressed cell layers or detached tumor cell groups at the advancing edge of each tumor. Staining intensity was scored into 3 grades: 0 , none and very weak (- and \pm$)$; 1 , weakly positive $(+)$; and 2 strongly positive (++) (Fig. 1). The percentage of stained cells (positive frequency) was scored into 4 grades: $1, \leq 25 \% ; 2,26-\leq 50 \% ; 3$, $51-\leq 75 \%$; and $4, \geq 76 \%$ cells. Composite scores were derived by addition of the intensity score and positive frequency score for statistical analysis with respect to each patient. A composite score of 6 was defined as high expression, and scores $\leq 5$ were defined as low expression. Any discrepant evaluations were re-examined simultaneously by the two investigators and a pathologist at the Tokyo Medical and Dental University using a double-headed light microscope (BX53; Olympus Corporation, Tokyo, Japan) and one monitor to achieve consensus.
Statistical analysis. The $\chi^{2}$ test was used to test possible associations between PAK5 expression and clinicopathological factors. Kaplan-Meier curves were plotted to assess the effect of PAK5 expression on disease-specific survival (DSS) and relapse-free interval (RFI). Survival curves were compared using the log-rank test. Multivariable Cox's proportional hazards regression models were used to assess the prognostic significance of PAK5 expression and other clinicopathological factors. Statistical analysis was performed using IBM SPSS software (version 22.0; IBM, Armonk, NY, USA). P<0.05 was considered to indicate a statistically significant difference.

\section{Results}

Immunohistochemical analysis of PAK5 expression. PAK5 expression was primarily observed in the cytoplasm of tumor and non-tumor cells. Although expression was detected in 
Table I. Correlations between PAK5 expression and clinicopathological factors of patients with gastric cancer.

PAK5 expression

Clinicopathological factors

Patients, $\mathrm{n}$

Low $(\mathrm{n}=235)$

High $(n=44)$

P-value

\section{Age}

$\geq 70$ years

$<70$ years

110

169

Sex

Male

Female

Location
L, M

$\mathrm{U}$

213

66

224

55

129

150

116

163

T2/T3/T4

Lymph node metastasis

N0

144

135

N1/N2/N3

195

84

133

146

\section{Stage}

II/III/IV

92
143

143

174

61

192

43

97

138

110

125

132

103

170

65

122

113
18

26

39

5

32

12

32

12

6

38

12

32

25

19

11

33

2

0.826

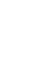

PAK5, P21-activated kinase 5. L, lower third of the stomach; M, middle third of the stomach; U, upper third of the stomach.

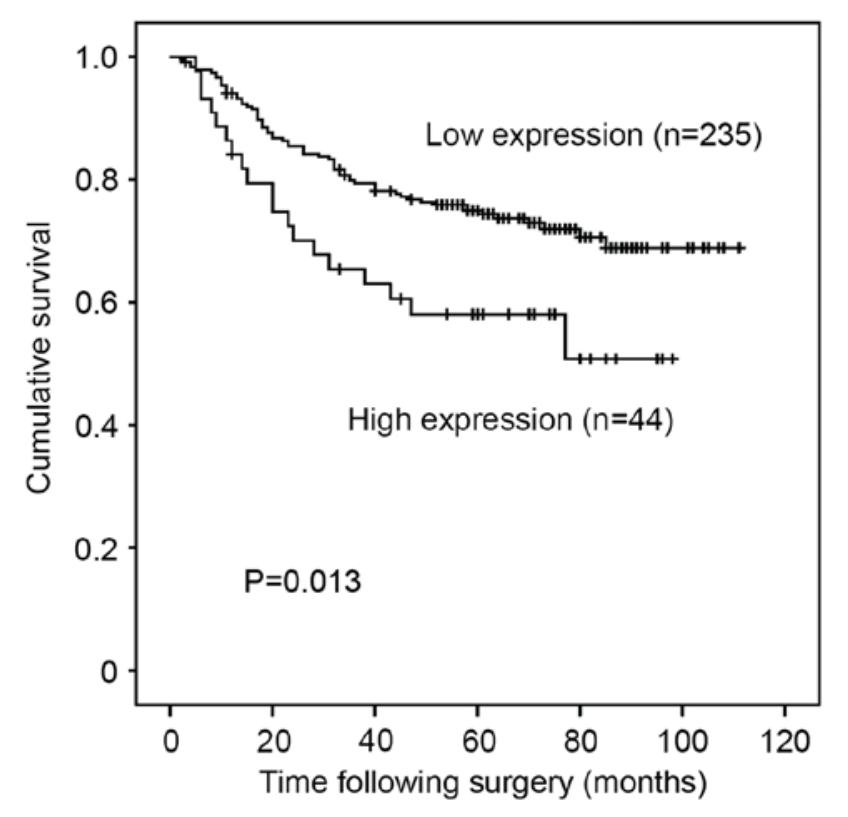

Figure 2. Kaplan-Meier curves for disease-specific survival of all patients with gastric cancer with expression of PAK5 showing reduced survival in patients with high expression. PAK5, P21-activated kinase 5. some nuclei of cancer cells, only cytoplasmic staining was counted towards a positive frequency score. Cancer cells were at least weakly stained in 257/279 (92\%) tumor samples. PAK5 staining was rarely detected in normal epithelium (Fig. 1). Metaplastic intestinal epithelium located close to cancer lesions was weakly, but never strongly stained. Almost all fibroblasts, smooth muscle and muscularis mucosae were uniformly stained. Of the 279 patients, 44 (15.8\%) exhibited high PAK5 expression. Complete absence of PAK5 staining in cancer cells was observed in 13 slides of poorly differentiated adenocarcinoma, 6 slides of signet-ring cell adenocarcinoma and 1 each for mucinous, papillary and tubular adenocarcinoma samples.

Association between PAK5 expression and clinicopathological variables. Correlations between the expression of PAK5 and clinicopathological factors are illustrated in Table I. High expression of PAK5 was significantly associated with the differentiated pathological type (differentiated vs. undifferentiated; $\mathrm{P}<0.001)$, depth of tumor invasion $(\mathrm{T} 1$ vs. T2-T4; $\mathrm{P}<0.001$ ), lymph node metastasis (N0 vs. N1-N3; $\mathrm{P}<0.001$ ), presence of distant metastasis or recurrence (present 
Table II. Prognostic factors in univariate and multivariate Cox's proportional hazard regression models for DSS.

\begin{tabular}{|c|c|c|c|c|c|}
\hline \multirow[b]{2}{*}{ Clinicopathological factors } & \multicolumn{2}{|c|}{ Univariate (Log-rank) } & \multicolumn{3}{|c|}{ Multivariate } \\
\hline & 5-year DSS (\%) & P-value & HR & $95 \% \mathrm{CI}$ & P-value \\
\hline \multicolumn{6}{|l|}{ Age } \\
\hline$\geq 70$ years & 67 & 0.100 & & & \\
\hline$<70$ years & 75.5 & & & & \\
\hline \multicolumn{6}{|l|}{ Sex } \\
\hline Male & 75.6 & 0.878 & & & \\
\hline Female & 67.1 & & & & \\
\hline \multicolumn{6}{|l|}{ Location } \\
\hline $\mathrm{L}, \mathrm{M}$ & 76.5 & 0.020 & 1 & & 0.029 \\
\hline $\mathrm{U}$ & 58.1 & & 1.74 & $1.06-2.85$ & \\
\hline \multicolumn{6}{|l|}{ Pathological type } \\
\hline Differentiated & 81.6 & 0.003 & 1 & & 0.447 \\
\hline Undifferentiated & 65 & & 1.21 & $0.74-2.00$ & \\
\hline \multicolumn{6}{|l|}{ Depth of invasion } \\
\hline $\mathrm{T} 1$ & 97.3 & $<0.001$ & 1 & & $<0.001$ \\
\hline $\mathrm{T} 2 / \mathrm{T} 3 / \mathrm{T} 4$ & 64.7 & & 6.60 & $2.29-18.99$ & \\
\hline \multicolumn{6}{|l|}{ Lymph node metastasis } \\
\hline Negative (N0) & 94.2 & $<0.001$ & 1 & & $<0.001$ \\
\hline Positive (N1/2/3) & 57.5 & & 5.26 & $2.51-11.04$ & \\
\hline \multicolumn{6}{|l|}{ Distant metastasis } \\
\hline Negative & 79.4 & $<0.001$ & 1 & & $<0.001$ \\
\hline Positive & 0.0 & & 5.79 & $3.42-9.81$ & \\
\hline \multicolumn{6}{|l|}{ PAK5 } \\
\hline Low & 74.8 & 0.014 & 1 & & 0.659 \\
\hline High & 58 & & 1.14 & $0.65-2.00$ & \\
\hline
\end{tabular}

DSS, disease specific survival; PAK5, P21-activated kinase 5; HR, hazard ratio; CI, confidence interval. L, lower third of the stomach; M, middle third of the stomach; $\mathrm{U}$, upper third of the stomach.

vs. absent; $\mathrm{P}=0.038$ ), and advanced tumor stage (I vs. II-IV; $\mathrm{P}=0.001)$. None of the 30 signet-ring cell adenocarcinoma slides exhibited high PAK5 expression.

Association between DSS and RFI. High expression of PAK5 was significantly associated with poorer DSS ( $\mathrm{P}=0.013$; Fig. 2). The 5-year DSS rate was $58.0 \%$ in patients with high expression of PAK 5 and $74.8 \%$ in those with low expression. Upper stomach lesion, undifferentiated type of cancer, depth of invasion of tumor, positive lymph node metastases, positive distant metastases, advanced pathological stages and high expression of PAK5 were significantly associated with poorer DSS following univariate analysis (Table II). However, high expression of PAK5 was not an independent prognostic factor [hazard ratio $(\mathrm{HR})=1.14 ; 95 \%$ confidence interval $(\mathrm{CI}), 0.65-2.00 ; \mathrm{P}=0.659]$ following multivariate Cox's proportional hazards regression analysis adjusted for the following clinical prognostic factors: Localization of tumor; histopathological type; depth of invasion; lymph node metastases; and distant metastases (Table II).

For patients with stage I to III disease $(n=254)$, high PAK5 expression was significantly associated with poorer RFI on univariate analysis ( $\mathrm{P}=0.044$; Fig. 3). However, following multivariate analysis adjusted for location of the tumor, histopathology, depth of invasion and lymph node metastases, PAK5 expression was not an independent prognostic factor for RFI (HR=0.96; 95\% CI, 0.48-1.91; $\mathrm{P}=0.909)$ (Table III).

\section{Discussion}

The present study demonstrated that high expression of PAK5 was significantly associated with depth of tumor invasion, lymph node metastasis, distant metastasis and recurrence in patients with gastric cancer. Furthermore, high expression of PAK5 was revealed to be significantly associated with poorer DSS and RFI. However, high expression of PAK5 was not identified as an independent prognostic factor following multivariate analysis. A previous study that investigated the potential associations between PAK5 and gastric cancer reported that none of the clinicopathological parameters were associated to PAK5 expression (13). This discrepancy may be associated with the different scoring systems and methodologies used to measure PAK5 status. In the current study, no PAK5 staining was detected in normal epithelium, and metaplastic intestinal epithelium located close to cancer 
Table III. Prognostic factors in univariate and multivariate Cox's proportional-hazards regression models for RFI in stage I to III.

\begin{tabular}{|c|c|c|c|c|c|}
\hline \multirow[b]{2}{*}{ Clinicopathological factor } & \multicolumn{2}{|c|}{ Univariate (Log-rank) } & \multicolumn{3}{|c|}{ Multivariate } \\
\hline & 5-year RFI (\%) & P-value & HR & $95 \% \mathrm{CI}$ & P-value \\
\hline \multicolumn{6}{|l|}{ Age } \\
\hline$\geq 70$ years & 76 & 0.833 & & & \\
\hline$<70$ years & 77.2 & & & & \\
\hline \multicolumn{6}{|l|}{ Sex } \\
\hline Male & 75 & 0.725 & & & \\
\hline Female & 76.5 & & & & \\
\hline \multicolumn{6}{|l|}{ Location } \\
\hline $\mathrm{L}, \mathrm{M}$ & 81.1 & 0.002 & 1 & & 0.014 \\
\hline $\mathrm{U}$ & 59.3 & & 2.00 & $1.15-3.46$ & \\
\hline \multicolumn{6}{|l|}{ Pathological type } \\
\hline Differentiated & 84.8 & 0.003 & 1 & & 0.310 \\
\hline Undifferentiated & 69.4 & & 1.38 & $0.74-2.55$ & \\
\hline \multicolumn{6}{|l|}{ Depth of invasion } \\
\hline $\mathrm{T} 1$ & 96.5 & $<0.001$ & 1 & & 0.001 \\
\hline $\mathrm{T} 2 / \mathrm{T} 3 / \mathrm{T} 4$ & 61.7 & & 6.30 & $2.20-18.06$ & \\
\hline \multicolumn{6}{|l|}{ Lymph node metastasis } \\
\hline Negative (N0) & 95.0 & $<0.001$ & 1 & & $<0.001$ \\
\hline Positive (N1/2/3) & 54.6 & & 6.92 & $3.02-15.84$ & \\
\hline \multicolumn{6}{|l|}{ PAK5 } \\
\hline Low & 79.3 & 0.044 & 1 & & 0.909 \\
\hline High & 65.4 & & 0.96 & $0.48-1.91$ & \\
\hline
\end{tabular}

RFI, relapse-free interval; PAK5, P21-activated kinase 5; HR, hazard ratio; CI, confidence interval. L, lower third of the stomach; M, middle third of the stomach; $\mathrm{U}$, upper third of the stomach.

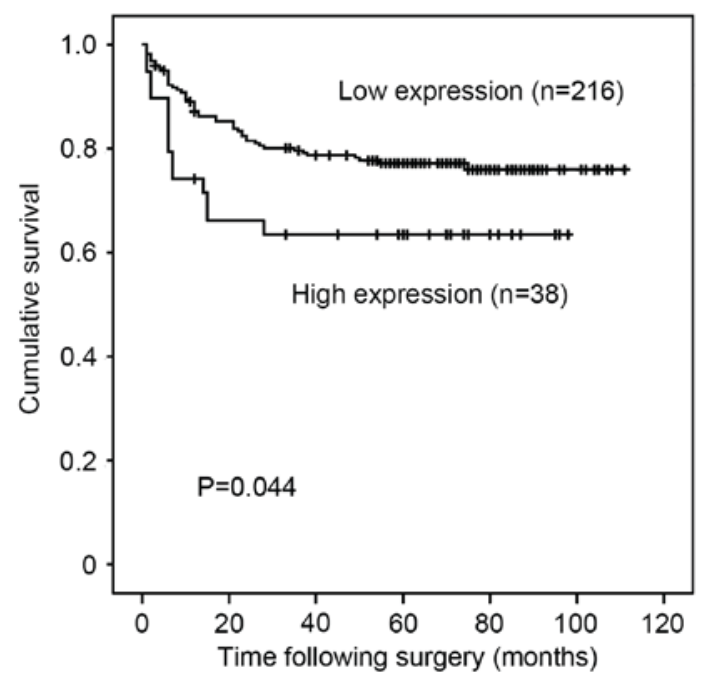

Figure 3. Kaplan-Meier curves for relapse-free interval of patients with stage I-III gastric cancer with expression of PAK5 showing reduced relapse-free interval in patients with high expression. PAK5, P21-activated kinase 5.

lesions was weakly stained in some cases, but never strongly stained. In this respect, the data of the present study are similar to the findings of $\mathrm{Gu}$ et al (13), who reported that the immunohistochemical expression level of PAK5 was significantly higher in cancer tissue compared with adjacent normal tissue samples. Overall, these findings remain consistent with an important role for PAK5 in gastric cancer progression.

PAK5 has been reported to be expressed predominantly in the brain, and to promote neurite outgrowth by interacting with GTPases, cell division control protein 42 homolog (Cdc42) and Rac, in a signaling pathway that is antagonistic to Rho $(9,17)$. In the human brain, PAK 5 contributes to microtubule stability by inactivating serine/threonine-protein kinase MARK (MARK) through binding to the catalytic domain, consequently preventing the MARK-induced phosphorylation of Tau $(6,18)$. The sub-cellular localization of PAK5 appears to be associated with some of its functions. The presence of the Cdc42/Rac interactive binding domain within PAK5 prevents its accumulation in the nucleus, and PAK5 binds to the Rho family small $\mathrm{G}$ proteins, RhoD and RhoH, in addition to $\mathrm{Cdc} 42$ (19). The interactions of PAK5 with Cdc42 and RhoD target it to different compartments within the cell (19). Endogenous PAK5 shuttles between mitochondria and the nucleus, and induces resistance to apoptosis by phosphorylating Bcl2-associated agonist of cell death in the mitochondria $(20,21)$. The role of PAK5 in the resistance to apoptosis may underlie our results hypothesizing that PAK5 may be involved in cancer metastasis. 
As is the case with numerous cancers, heterogeneity is prevalent in gastric cancer tissue. Despite this, certain proteins that are heterogeneously expressed in gastric cancer cells, including erb-b2 receptor tyrosine kinase 2 (HER2), are targets of clinically effective anticancer drugs (22). Only 10-20\% of all patients with gastric cancer overexpress HER2 $(23,24)$, and HER2 expression differs significantly by histological subtype with a high correlation between HER 2 expression and intestinal histologic type (23). In the present study, similar to HER2, PAK5 expression differed significantly by histological type, although some tumors contained differentiated and undifferentiated cancer cells, consistent with a complex underlying molecular pathogenesis that is at present poorly understood. Although several effective and specific small molecule pan-PAK inhibitors, or inhibitors of group I or II PAKs, particularly PAK1 and PAK4, are in advanced stages of preclinical testing (25), the development and efficacy of PAK5-specific inhibitors has not yet been reported.

In conclusion, high expression of PAK5 was significantly associated with differentiated pathological type, depth of tumor invasion, lymph node metastasis, presence of distant metastasis or recurrence, and therefore with advanced tumor stage, and poorer DSS and RFI rates. Overall, the data of the current study suggest that PAK5 is a potential drug target in gastric cancer.

\section{Acknowledgements}

The authors would like to thank Dr Takumi Akashi, pathologist at the Tokyo Medical and Dental University, for his advice and great help in the interpretation of the immunohistochemical data.

\section{References}

1. Global Burden of Disease Cancer Collaboration, Fitzmaurice C, Dicker D, Pain A, Hamavid H, Moradi-Lakeh M, MacIntyre MF, Allen C, Hansen G, Woodbrook R, et al: The global burden of cancer. JAMA Oncol 1: 505-527, 2015.

2. Lordick F, Lorenzen S, Yamada Y and Ilson D: Optimal chemotherapy for advanced gastric cancer: Is there a global consensus? Gastric Cancer 17: 213-225, 2014.

3. Kim C, Mulder K and Spratlin J: How prognostic and predictive biomarkers are transforming our understanding and management of advanced gastric cancer. Oncologist 19: 1046-1055, 2014.

4. Kumar R, Gururaj AE and Barnes CJ: p21-activated kinases in cancer. Nat Rev Cancer 6: 459-471, 2006.

5. Ye DZ and Field J: PAK signaling in cancer. Cell Logist 2: 105-116, 2012.

6. Wells CM and Jones GE: The emerging importance of group II PAKs. Biochem J 425: 465-473, 2010.

7. Fang ZP, Jiang BG, Gu XF, Zhao B, Ge RL and Zhang FB: $\mathrm{P} 21$-activated kinase 5 plays essential roles in the proliferation and tumorigenicity of human hepatocellular carcinoma. Acta Pharmacol Sin 35: 82-88, 2014.
8. Zhao ZS and Manser E: PAK and other Rho-associated kinases-effectors with surprisingly diverse mechanisms of regulation. Biochem J 386: 201-214, 2005.

9. Pandey A, Dan I, Kristiansen TZ, Watanabe NM, Voldby J, Kajikawa E, Khosravi-Far R, Blagoev B and Mann M: Cloning and characterization of PAK5, a novel member of mammalian p21-activated kinase-II subfamily that is predominantly expressed in brain. Oncogene 21: 3939-3948, 2002.

10. Kobayashi K, Inokuchi M, Takagi Y, Otsuki S, Fujimori Y, Sato Y, Yanaka Y, Higuchi K, Aburatani T, Tomii C, et al: Prognostic significance of PAK4 expression in gastric cancer. J Clin Pathol 69: 580-585, 2016.

11. Giroux V, Iovanna J and Dagorn JC: Probing the human kinome for kinases involved in pancreatic cancer cell survival and gemcitabine resistance. FASEB J 20: 1982-1991, 2006.

12. Gong W, An Z, Wang Y, Pan X, Fang W, Jiang B and Zhang H: P21-activated kinase 5 is overexpressed during colorectal cancer progression and regulates colorectal carcinoma cell adhesion and migration. Int J Cancer 125: 548-555, 2009.

13. Gu J, Li K, Li M, Wu X, Zhang L, Ding Q, Wu W, Yang J, Mu J, Wen $\mathrm{H}$, et al: A role for p21-activated kinase 7 in the development of gastric cancer. FEBS J 280: 46-55, 2013.

14. Gu X, Wang C, Wang X, Ma G, Li Y, Cui L, Chen Y, Zhao B and Li K: Efficient inhibition of human glioma development by RNA interference-mediated silencing of PAK5. Int J Biol Sci 11: 230-237, 2015.

15. Japanese Gastric Cancer Association: Japanese classification of gastric carcinoma: 3rd English edition. Gastric Cancer 14: 101-112, 2011.

16. International Union Against Cancer (UICC): TNM classification of malignant tumors. Sobin LH, Gospodarowicz MK and Wittekind C (eds). 7th edition. Wiley-Blackwell, Oxford 2009.

17. Dan C, Nath N, Liberto $M$ and Minden A: PAK5, a new brain-specific kinase, promotes neurite outgrowth in N1E-115 cells. Mol Cell Biol 22: 567-577, 2002.

18. Timm T, Matenia D, Li XY, Griesshaber B and Mandelkow EM: Signaling from MARK to tau: Regulation, cytoskeletal crosstalk, and pathological phosphorylation. Neurodegener Dis 3: 207-217, 2006.

19. Wu X and Frost JA: Multiple Rho proteins regulate the subcellular targeting of PAK5. Biochem Biophys Res Commun 351: 328-335, 2006.

20. Cotteret S, Jaffer ZM, Beeser A and Chernoff J: p21-activated kinase 5 (Pak5) localizes to mitochondria and inhibits apoptosis by phosphorylating BAD. Mol Cell Biol 23: 5526-5539, 2003.

21. Cotteret S and Chernoff J: Nucleocytoplasmic shuttling of Pak5 regulates its antiapoptotic properties. Mol Cell Biol 26: 3215-3230, 2006.

22. Bang YJ, Van Cutsem E, Feyereislova A, Chung HC, Shen L, Sawaki A, Lordick F, Ohtsu A, Omuro Y, Satoh T, et al: Trastuzumab in combination with chemotherapy versus chemotherapy alone for treatment of HER2-positive advanced gastric or gastro-oesophageal junction cancer (ToGA): A phase 3, open-label, randomised controlled trial. Lancet 376: 687-697, 2010.

23. Gravalos C and Jimeno A: HER2 in gastric cancer: A new prognostic factor and a novel therapeutic target. Ann Oncol 19: 1523-1529, 2008.

24. Hofmann M, Stoss O, Shi D, Büttner R, van de Vijver M, Kim W, Ochiai A, Rüschoff J and Henkel T: Assessment of a HER2 scoring system for gastric cancer: Results from a validation study. Histopathology 52: 797-805, 2008.

25. Radu M, Semenova G, Kosoff R and Chernoff J: PAK signalling during the development and progression of cancer. Nat Rev Cancer 14: 13-25, 2014. 